\title{
Clinical outcomes of muscle invasive bladder Cancer according to the BASQ classification
}

\author{
Hyeong Dong Yuk', Chang Wook Jeong ${ }^{2}$, Cheol Kwak², Hyeon Hoe Kim², Kyung Chul Moon ${ }^{3+}$ and \\ Ja Hyeon $\mathrm{Ku}^{2^{*}+}$ (D)
}

\begin{abstract}
Background: We evaluated the clinical efficacy and prognosis of muscle-invasive bladder cancer according to the basal/squamous-like (BASQ) classification system based on immunohistochemical staining [CK5/6(+), CK14(+), $\operatorname{GATA3}(-)$, and FOXA1(-)].

Methods: One hundred patients diagnosed with muscle-invasive bladder cancer (cT2-4 N0-3 M0) were included in the study. All patients underwent radical cystectomy after transurethral removal of bladder tumor. Immunostaining was performed for CK5/6, CK14, FOXA1, and GATA3 antibodies on tissue microarray slides, and expression patterns were quantitatively analyzed using a scanning program.

Results: The median follow-up time was 77.4 (interquartile range: 39-120.9) months. The mean age of the patients was $65.1 \pm 11.2$ years. FOXA1 or CK14 expression greater than $1 \%$ was respectively positively and negatively correlated with overall survival (OS; $p=0.011$ and $p=0.042$, respectively), cancer-specific survival (CSS; $p=0.050$ for both), and recurrence-free survival (RFS; $p=0.018$ and $p=0.040$, respectively). For CK5/6+ and GATA3- or FOXA1expression, $10 \% \mathrm{CK} 5 / 6+$ cells were negatively correlated with OS ( $p=0.032$ and $p=0.039$, respectively) and with RFS in combination with FOXA1- only $(p=0.050)$.

Conclusions: In this study, CK14 expression was associated with a poor prognosis. The new classification system of bladder cancer based on molecular characteristics is expected to helpful tool for the establishment of personalized treatment strategies and associated prediction of therapeutic responses.
\end{abstract}

Keywords: Basal cell, Immunohistochemistry, Molecular subtype, Neoplasm metastasis, Squamous cell, Urinary bladder neoplasms

\section{Background}

Bladder cancer is the fourth most common cancer in men, with approximately 60,000 new diagnoses each year [1], ranking as the eighth leading cause of cancerrelated deaths in the United States, with about 12,000 deaths annually [1]. Specifically, in 2017, there were 79, 030 cases of bladder cancer and 16,870 related deaths in the United States [1]. Approximately $90-95 \%$ of all bladder cancer cases are urothelial cell carcinoma, with the

\footnotetext{
* Correspondence: kuuro70@snu.ac.kr

†Kyung Chul Moon and Ja Hyeon Ku contributed equally to this work. ${ }^{2}$ Department of Urology, Seoul National Univervity College of Medicine, Seoul National University Hospital, Seoul, Korea

Full list of author information is available at the end of the article
}

minority consisting of non-urothelial cell carcinoma. During initial diagnosis, $70-80 \%$ of bladder cancers are diagnosed as non-invasive with the remaining 20-30\% diagnosed as invasive. Most cases of non-invasive bladder cancer can be treated with transurethral removal of the bladder tumor (TURB) alone [2, 3]. However, a high recurrence rate after TURB has been reported within 1 year $(15-70 \%)$ and 5 years $(7-40 \%)$ [2, 3]. Therefore, continuous additional testing and repeated treatments are often needed. Indeed, in the United States, bladder cancer is reportedly one of the tumors for which patients incur a high costof $[2,3]$.

Recently, a large-scale, detailed analysis of the molecular genetic characteristics of bladder cancer was reported

(c) The Author(s). 2019 Open Access This article is distributed under the terms of the Creative Commons Attribution 4.0 International License (http://creativecommons.org/licenses/by/4.0/), which permits unrestricted use, distribution, and 
through The Cancer Genome Atlas (TCGA) [2, 3]. The TCGA study revealed that bladder cancer can be classified into several subtypes depending on the molecular characteristics of the genomes [4-9]: luminal type, basal type, p53-like tumor, and small cell carcinoma-like tumor. Among these subtypes, the basal type is associated with a particularly poor prognosis [4-9]. Moreover, the basal type and p53-like tumor are highly resistant to preoperative chemotherapy; thus, identifying the accurate subtype is an essential factor in clinical decisionmaking [5]. Basal/squamous-like (BASQ) is a basal type of bladder cancer with a very poor prognosis and high rate of resistance to chemotherapy [5]. It is immunohistochemically defined by CK5/6(+), CK14(+), GATA3(-) and FOXA1(-) expression [5]. However, there is no report on the treatment response and prognosis of patients with bladder cancer when applying this new classification system. Therefore, in the present study, we evaluated the clinical efficacy and prognosis of MIBC according to the use of the BASQ classification system in clinical practice.

\section{Methods}

\section{Ethics}

This study was approved by the Institutional Review Board (IRB No. H-1806-081-951). We used the human bladder cancer materials stored in the cancer tissue bank (IRB No. H1307-084-505). We obtained informed consent from all research participants.

\section{Patient populations}

A total of 100 patients with muscle-invasive urothelial carcinoma (cT2-4 N0-3 M0) of the urinary bladder were included in the study. Patient selection was based on the availability of sufficient material for immunohistochemistry. All patients underwent TURB followed by radical cystectomy between 2000 and 2012 at Seoul National University Hospital.

\section{Tissue microarray (TMA) construction}

Hematoxylin and eosin slides were reviewed for confirmation of the pathologic diagnosis and various pathologic parameters, including invasion depth and grade. We constructed TMA blocks from formalin-fixed paraffinembedded tissue blocks (Superbiochips Laboratories, Seoul, Korea). In brief, two representative tumor cores ( $2 \mathrm{~mm}$ in diameter) were selected from the viable tumor area. The cancer tissues of patients were examined microscopically by a skilled pathologist, and the TMA was prepared after selecting the most representative cancer tissues. Immunostaining was performed for CK5/6, CK14, FOXA1, and GATA3 antibodies on TMA slides from the 100 patient samples, and the expression patterns were quantitatively analyzed using a scanning program. Based on the expression patterns, the patients were divided according to the BASQ classification (CK5/ 6, CK14, FOXA1, and GATA3).

The prognostic value of the BASQ classification was determined based on clinical and pathological information such as age, body mass index, sex, American Society of Anesthesiologists (ASA) physical status, pathologic TNM stage, carcinoma in situ status, lymphovascular invasion, margin-positive status, lymph node dissection range, number of removed lymph nodes, number of positive lymph nodes, and neoadjuvant chemotherapy enforcement. We also collected various types of oncological data, including the recurrence, mortality, and cancer-related mortality rates.

\section{Immunohistochemistry (IHC)}

IHC staining was performed on 4- $\mu$ m-thick sections from TMA blocks using the Benchmark XT autostainer (Ventana Medical Systems, Tucson, AZ, USA). The sections were incubated with the following primary antibodies: mouse monoclonal antibodies against CK5/6 (64 min; 1:50; Dako, Glostrup, Denmark), CK14 (32 min; 1: 50; Cell Marque, Rocklin, CA, USA), and GATA3 (32 min; 1:500; clone 156-3C11; Cell Marque), and rabbit polyclonal antibody against FOXA1 (16 min; 1:700; ThermoFisher Scientific, Rockford, IL, USA). To interpret the IHC results, the percentage of positively stained tumor cells was semi-quantitatively evaluated into three categories; 0 , no positive cells; $1+, 1-10 \%$ positive cells; $2+, 11-25 \%$ positive cells; $3+,>25 \%$ positive cells.

\section{Statistical analysis}

Continuous variables are presented as the median value and interquartile ranges (IQRs) or average value and standard deviations (SDs). Nominal variables are presented as the frequency of events (\%). The primary endpoint of the study was the overall survival (OS) rate, and the secondary endpoints were cancer-specific survival (CSS) and recurrence-free survival (RFS). The KaplanMeier method was used to predict all survival outcomes, and significance among groups was determined using log-rank tests. Cox proportional hazards regression analysis was used for analysis of various oncology outcomes and predictors. All statistical tests were performed using IBM SPSS Statistics version 22.0 (IBM, Armonk, NY, USA) and STATA version 14 (StataCorp LP, College Station, Texas). A $p$-value $<0.05$ was considered statistically significant.

\section{Results}

Baseline characteristics of the patients

Table 1 shows the basic characteristics of the $100 \mathrm{pa}$ tients involved in the study. The median follow-up time was 77.4 (IQR: 39-120.9) months. The mean age of the 
Table 1 Basic patient characteristics

\begin{tabular}{|c|c|}
\hline Variable & $N=100$ \\
\hline Age (mean $\pm S D)$ (year) & $65.1 \pm 11.2$ \\
\hline BMI (m2/kg) & $23.56 \pm 5.93$ \\
\hline \multicolumn{2}{|l|}{ Gender } \\
\hline Female & $17(17.0 \%)$ \\
\hline Male & $83(83.0 \%)$ \\
\hline \multicolumn{2}{|l|}{ ASA } \\
\hline 1 & $34(34.0 \%)$ \\
\hline 2 & $57(57.0 \%)$ \\
\hline$\geq 3$ & $9(9.0 \%)$ \\
\hline \multicolumn{2}{|l|}{ pT stage } \\
\hline $\mathrm{T} 2$ & $50(50.0 \%)$ \\
\hline T3 & $42(42.0 \%)$ \\
\hline T4 & $8(8.0 \%)$ \\
\hline LVI & $42(42.0 \%)$ \\
\hline $\mathrm{ClS}$ & $34(34.0 \%)$ \\
\hline \multicolumn{2}{|l|}{ N stage } \\
\hline NO & $80(80.0 \%)$ \\
\hline N1 & $7(7.0 \%)$ \\
\hline N2 & $8(8.0 \%)$ \\
\hline N3 & $5(5.0 \%)$ \\
\hline \multicolumn{2}{|l|}{ LND range } \\
\hline Standard & $36(36.0 \%)$ \\
\hline Extend & $64(64.0 \%)$ \\
\hline Removed LN & $14.1 \pm 12.9$ \\
\hline Positive LN & $1.0 \pm 2.8$ \\
\hline Recurrence & $31(31.0 \%)$ \\
\hline Mortality & $59(59.0 \%)$ \\
\hline Cancer related mortality & $32(32.0 \%)$ \\
\hline
\end{tabular}

BMI Body mass index, LVI Lymphovascular invasion, CIS Carcinoma in situ, LND Lymph node dissection, LN Lymph node;

patients was $65.1 \pm 11.2$ years, and more than $80 \%$ of the patients were males. Ninety-one patients (91\%) had an ASA physical status below 3. All patients were diagnosed as having muscle-invasive bladder cancer with T2-4 N0$3 \mathrm{M0} ; 10 \%$ of the patients underwent neoadjuvant chemotherapy, $35 \%$ of the patients underwent radical cystectomy with standard pelvic lymph node dissection (PLND), whereas $65 \%$ of the patients had extended PLND. Moreover, $65 \%$ of the patients underwent ileal conduit urinary diversion, and the remaining 35\% underwent neobladder diversion.

Prognostic significance of FOXA1, GATA3, CK14, and CK5/ 6 expression

Table 2 shows semi-quantitatively evaluated $\mathrm{IHC}$ results. CK5/6 and CK14 staining showed membranous expression, and GATA3 and FOXA1 staining was present in the nucleus (Fig. 1). A frequency of FOXA1 expression greater than $1 \%$ was positively correlated with OS $(p=0.011)$, CSS $(p=0.050)$, and RFS $(p=0.018)$ (Fig. 2). In addition, a FOXA1 positive frequency greater than $10 \%$ was positively correlated with CSS $(p=0.022)$, and a frequency above $25 \%$ was positively correlated with RFS ( $\mathrm{p}=0.011)$.

OS, CSS, and RFS all tended to improve in patients with $\geq 1 \%$ GATA3 expression compared to those with < $1 \%$ expression, although the difference was not statistically significant (Fig. 2). GATA3 expression greater than $10 \%$ was positively correlated with RFS $(p=0.032)$.

A CK14 expression rate greater than $1 \%$ was negatively correlated with OS $(p=0.042)$, CSS $(p=0.050)$, and RFS $(p=0.040)$ (Fig. 2). Similarly, OS and RFS tended to be worse in patients with $\geq 1 \%$ CK5/6 expression than in patients with $<1 \%$ CK5/6 expression but not significantly (Fig. 2). However, CSS was better in patients with $<1 \%$ CK5/6 expression than in those with $\geq 1 \%$ expression $(p=0.028)$.

Additional file 1 shows nomogram for predictors of survival after cystectomy. In multivariable Cox regression analysis, OS was significantly correlated with the expression of CK14 (HR: 6.16, 95\% CI: 1.28-38.30) and FOXA1 (HR: 0.08, 95\% CI: 0.01-0.59) in the urothelial carcinoma subtype (Table 3). In CSS, expression of CK14 (HR: 3.96, 95\% CI: 1.13-16.36) and FOXA1 (HR: 0.08, 95\% CI: 0.01-0.61) was also significantly correlated. CK14 was negatively correlated with OS and CSS, and FOXA1 was positively correlated with OS and CSS (Table 3). In RFS, only CK14 was negatively correlated with RFS (HR: 3.19, 95\% CI: 1.07-9.55).

A comparison of oncologic outcomes between the $<1 \%$, $1-10 \%, 11-25,>25 \%$ groups showed that FOXA1 expression in the 1-10\% group was positively correlated with OS compared to that in less than $1 \%$; OS $(p=0.007)$, CSS $(p=0.001)$, and RFS group $(p=0.025)$ (Fig. 3). CK14 was negatively correlated with OS, CSS, and RFS according to subtype expression level. A comparison of oncologic outcomes showed that in both the lesser than $1 \%$ and between 11 and 25\% groups, CK14 expression between 11 and $25 \%$, was negatively correlated with OS compared to that in lesser than 1\%; OS ( $\mathrm{p}=0.001)$, CSS $(\mathrm{p}=0.001)$, and RFS ( $p=0.004)$ (Fig. 3). A comparison of oncologic outcomes between the 1 and $10 \%$ and between 11 and $25 \%$ groups showed that CK14 expression in the between 11 and $25 \%$ group was negatively correlated with OS compared to that in the lesser than $1 \%$; OS $(p=0.002)$, CSS $(\mathrm{p}=0.001)$, and RFS group $(p=0.003)$ (Fig. 3).

\section{Relationship between basal type and prognosis}

In the case of CK5/6+ and GATA3- samples, more than $1 \%$ CK5/6 expression and GATA3- expression was significantly 
Table 2 Multivariable Cox regression analysis of overall survival, cancer specific survival, recurrence free survival

\begin{tabular}{|c|c|c|c|c|c|c|}
\hline \multirow[t]{2}{*}{ Parameter } & \multicolumn{2}{|l|}{ Overall survival } & \multicolumn{2}{|c|}{ Cancer specific survival } & \multicolumn{2}{|c|}{ Recurrence free survival } \\
\hline & $\mathrm{HR}(95 \% \mathrm{Cl})$ & $P$-value & $\mathrm{HR}(95 \% \mathrm{Cl})$ & $P$-value & $\mathrm{HR}(95 \% \mathrm{Cl})$ & $P$-value \\
\hline \multicolumn{7}{|l|}{ pT stage } \\
\hline $\mathrm{T} 2$ & reference & & reference & & Reference & \\
\hline$\geq \mathrm{T} 3$ & $1.46(1.15-1.85)$ & 0.002 & $1.49(1.17-1.90)$ & 0.001 & $2.04(0.38-10.74)$ & 0.400 \\
\hline LVI & $0.86(0.25-2.93)$ & 0.806 & $2.33(0.44-12.16)$ & 0.316 & $2.66(0.97-7.65)$ & 0.060 \\
\hline CIS & $0.90(0.28-2.89)$ & 0.858 & $4.15(1.21-16.03))$ & 0.028 & $0.70(0.43-1.14)$ & 0.160 \\
\hline N stage & & 0.478 & & 0.096 & & 0.096 \\
\hline No & reference & & Reference & & reference & \\
\hline$\geq N 1$ & $1.71(1.46-1.99)$ & $<0.001$ & $1.75(1.62-1.91)$ & 0.004 & $2.96(1.40-6.22)$ & 0.004 \\
\hline CK5/6 & $3.30(0.81-14.63)$ & 0.101 & $2.57(0.90-8.16)$ & 0.088 & $3.15(0.8-12.63)$ & 0.104 \\
\hline CK14 & $6.16(1.28-38.30)$ & 0.033 & $3.96(1.13-16.36)$ & 0.040 & $3.19(1.07-9.55)$ & 0.037 \\
\hline GATA3 & $0.77(0.37-1.58)$ & 0.477 & $0.81(0.22-2.89)$ & 0.742 & $0.29(0.01-5.27)$ & 0.409 \\
\hline FOXA1 & $0.08(0.01-0.59)$ & 0.023 & $0.08(0.01-0.61)$ & 0.024 & $0.12(0.1-1.51)$ & 0.103 \\
\hline
\end{tabular}

HR Hazard ratio, CI Confidence interval, LVI Lymphovascular invasion, CIS Carcinoma in situ, LND Lymph node dissection, LN Lymph node, UC Urothelial carcinoma

negatively correlated with OS $(p=0.032$; Fig. 4$)$. In the case of CK5/6+ and FOXA1- samples, more than $1 \%$ CK5/6+ expression and FOXA1 expression was significantly negatively correlated with OS and CSS $(p=0.039$ and $p=0.050$, respectively; Fig. 4). In the case of CK14+ and GATA3samples and CK14+ and FOXA1- samples were not significantly correlated with OS, CSS and RFS.

\section{Discussion}

Several recent studies have shown that in addition to the well-known Clinic factors, various antropometric factors have an effect on the outcome of the bladder cancer [10-13]. The recurrence rate of bladder cancer is reported to be significantly higher in obese patients than in normal weight patients $[10,13]$. Metabolic features such as obesity and associated insulin resistance have also been reported to affect prognosis [13]. High BMI also helps to predict poor prognosis, such as lymph node metastasis [10]. Immunological and inflammation markers such as basophil count, neutrophil and lymphocyte count, and C-reactive protein are also helpful in predicting recurrence after cystectomy or intravesical Bacillus Calmette-Guérin (BCG) treatment [11, 12].
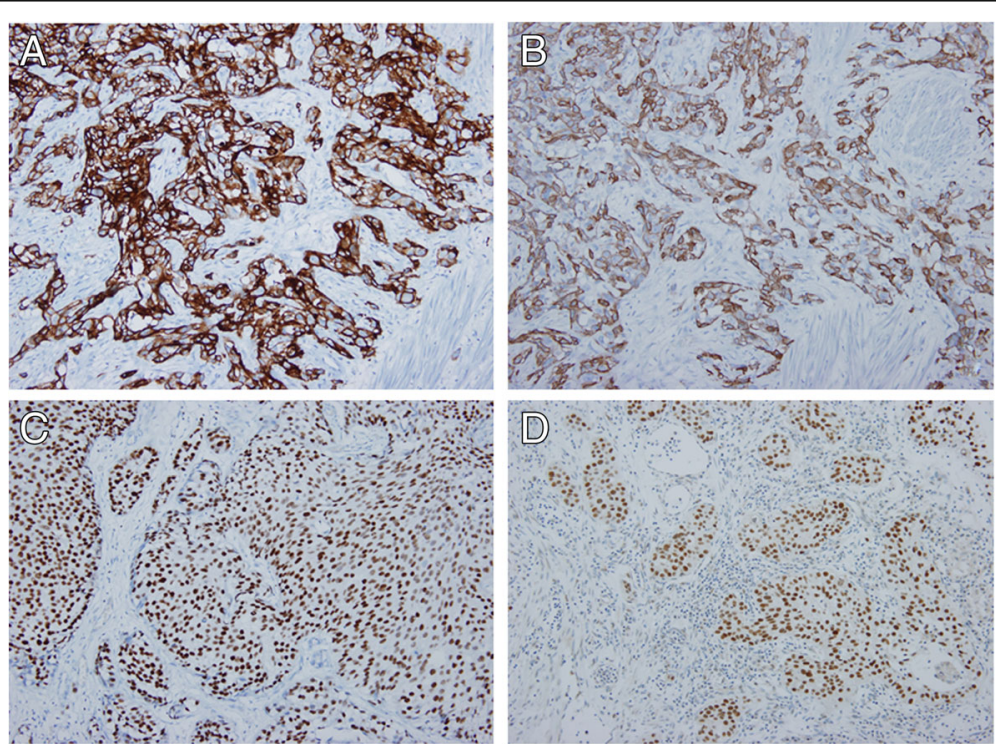

Fig. 1 Positive immunohistochemical staining of CK5/6 (a), CK14 (b), GATA3 (c), and FOXA1 (d). CK5/6, CK14 showed membranous staining, and GATA3, FOXA1 revealed nuclear positivity 


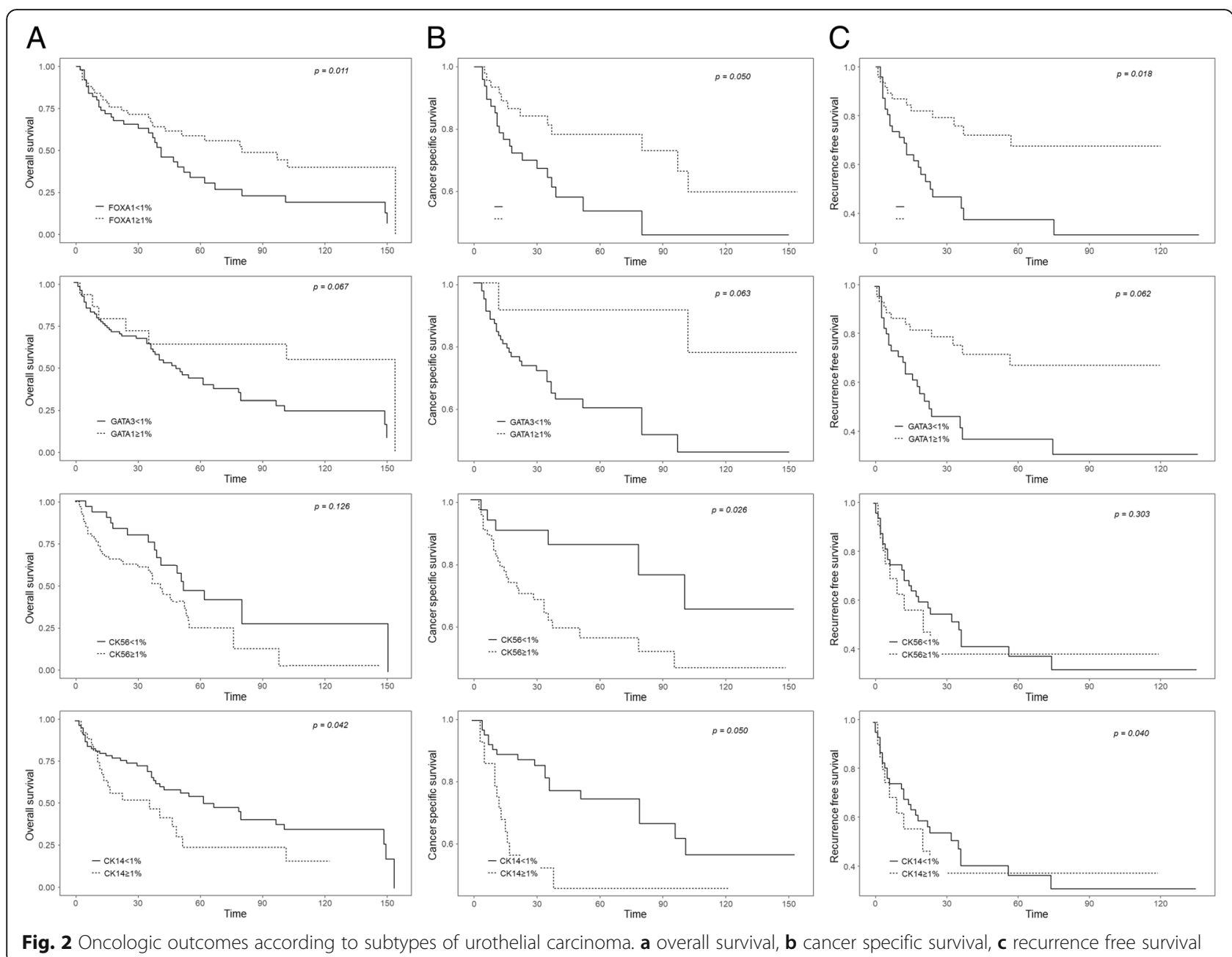

In addition to these various antropometric factors, histologic features have been reported to be helpful in predicting the prognosis of bladder cancer. Recent molecular studies have provided new insight into the factors contributing to bladder cancer development and progression. TMAs have been used to analyze genome expression, and immunohistochemical expression patterns are used to classify unique molecular types of bladder cancer. The gene mutations identified to date include genes related to chromatin regulation, cell cycle regulation, and kinase signaling pathways. In particular, molecular insight has been gained with respect to the cell and molecular biology of the urothelium, with 32 gene mutations significantly and

Table $\mathbf{3}$ Immunohistochemistry results

\begin{tabular}{lllll}
\hline & CK14 & CK5/6 & GATA3 & FOXA1 \\
\hline$<1 \%$ & $60(60.0 \%)$ & $24(24.0 \%)$ & $8(8.0 \%)$ & $15(15.0 \%)$ \\
$1 \sim 10 \%$ & $12(12.0 \%)$ & $35(35.0 \%)$ & $11(11.0 \%)$ & $15(15.0 \%)$ \\
$11 \sim 25 \%$ & $15(15.0 \%)$ & $16(16.0 \%)$ & $14(14.0 \%)$ & $20(20.0 \%)$ \\
$>25 \%$ & $13(13.0 \%)$ & $25(25.0 \%)$ & $67(67.0 \%)$ & $50(50.0 \%)$ \\
\hline
\end{tabular}

repeatedly observed in urothelial cell carcinoma, including genes related to cell cycle regulation, chromatin regulation, and kinase signaling pathways [5]. In particular, tumor protein 53 (TP53), fibroblast growth factor receptor-3 (FGFR3) mutations, and genes involved in the phosphatidylinositol-3-OH kinase (Pl3K)/protein kinase B (Akt)/mammalian target of rapamycin (mTOR) pathway were found to be associated with the prognosis of bladder cancer [5].

Besides the specific mutations and pathways, the discovery of molecular subtypes of urothelial cell carcinoma represents another important advance obtained through molecular studies. Several studies on genome expression profiles have reported that bladder cancer can be categorized into two intrinsic molecular types: luminal and basal, which are similar to those in breast cancer $[5,14,15]$. The molecular subtype of urothelial carcinoma is related to cell differentiation [16]. Basal type and luminal type are distinguished by keratin markers. The basal type has keratins representing the basal/stem-cell compartment, and the luminal type has keratins representing the umbrella cell layer 


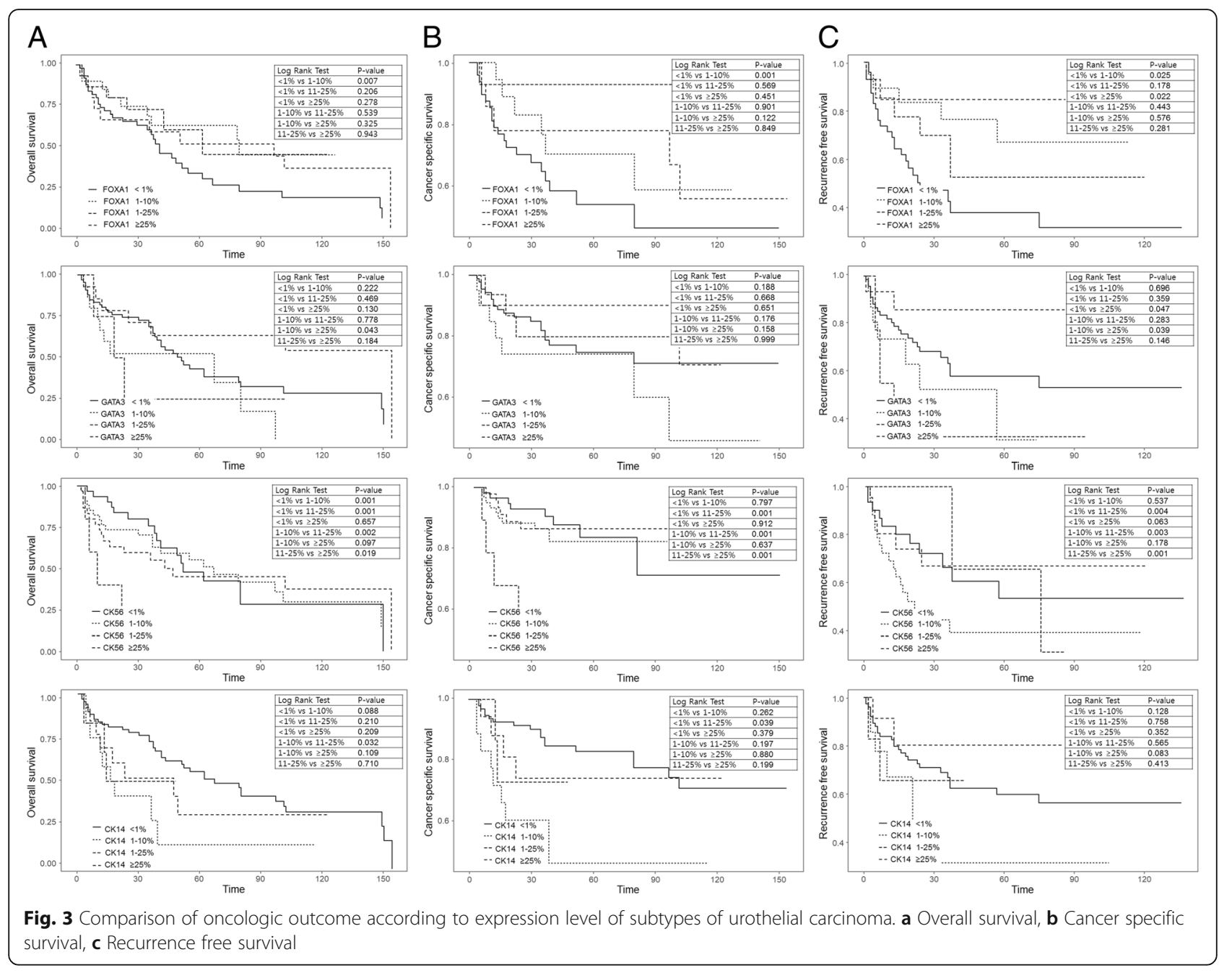

$[6,15]$. Basal type keratins are associated with the transcription factor $\triangle \mathrm{NP} 63$, which is related to a poor prognosis of muscle-invasive bladder cancer $[15,16]$.

Lindgren et al. [8] first classified samples from 144 patients with urothelial cell carcinoma according to gene expression patterns. They divided the urobasal group into two subgroups: urobasal A and B, according to their molecular characteristics. Urobasal A was mostly a nonmuscle invasive bladder cancer; however, patients with urobasal B showed a progressive phenotype with increased cell cycle activity and basal cell-related keratin expression [7].

The MD Anderson cohort was classified into basal and luminal types, which included 98 patients with invasive bladder cancers and 34 patients with superficial bladder cancers. The luminal type showed strong expression of markers such as CD24, FOXA1, GATA3, CK20, and XBP1, whereas the basal type was characterized by high-molecular-weight keratins (CK5 and CK14) and strong expression of $\mathrm{CDH} 3$ and $\mathrm{CD} 44[5,17]$.
Thus, the molecular characteristics of urothelial carcinoma can be used to predict the therapeutic effect and prognosis of the patient. McConkey et al.[18] reported that these molecular characteristics could predict the benefits of treatment such as chemotherapy or target agent therapy. Specifically, basal subtypes have been shown to be beneficial in neoadjuvant settings.[18].

Our present study also showed a tendency for a better prognosis in cancers with FOXA1 or GATA3 expression. Conversely, some of the CK14 and CK5/6-positive cases showed a tendency to be correlated with a poor prognosis. CK 14 negatively correlated with OS, CSS, and RFS, and FOXA1 positively correlated with OS and CSS. The expression of CK14 and FOXA1 subtypes seemed to be correlated with oncologic outcomes compared to those of CK56 and GATA3. Indeed, CK14 and FOXA1 expression may be a sensitive criterion for further differentiating urothelial carcinoma. However, our study was limited to 100 subjects and the results may be due to these limited subjects. 


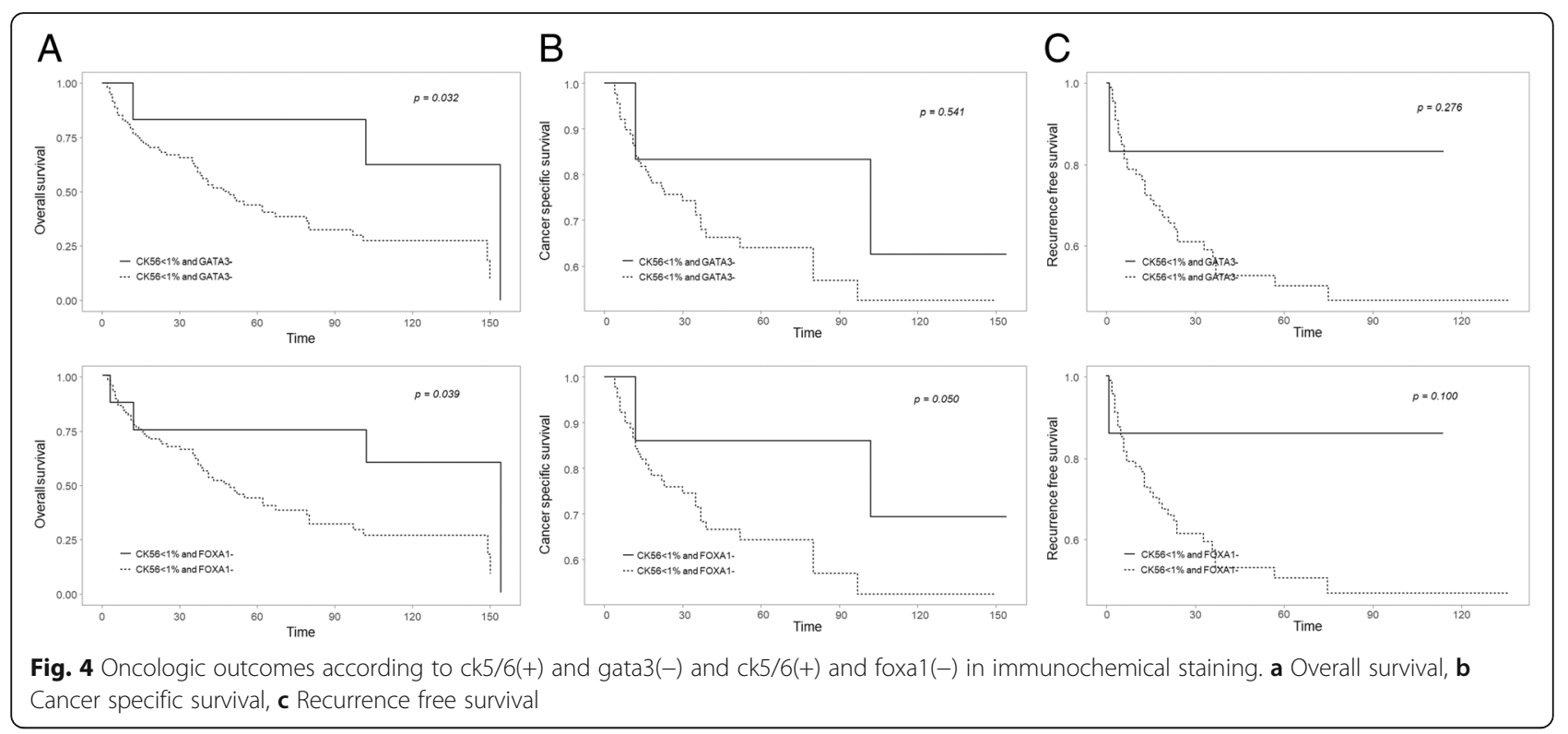

The difference in survival outcomes according to the degree of subtype expression was not significantly correlated with oncologic outcomes. However, CK14 and FOXA1 expression was correlated with oncologic outcomes at some yields. FOXA1 expression in the between 1 and 10\% group was positively correlated with OS compared to that in the lesser than $1 \%$ group, OS $(p=0.007)$, CSS $(p=0.001)$, and RFS ( $p=0.025)$ (Fig. 3).

CK14 also showed differences in oncologic outcome of OS and CSS according to subtype expression levels. There was a difference in the oncologic outcomes between the less than $1 \%$, between 11 and 25\%, and more than $25 \%$ groups. CK14 is negatively correlated with OS, CSS, and RFS according to subtype expression level (Fig. 3).

Even if FOXA1 is statistically significant in multivariate Cox regression analysis, the odd ratio is 0.08 and its impact is unclear. However, CK14 expression was associated with oncologic outcome of OS and CSS. (Table 3).

In the case of basal type cancers (CK14+, CK5/6+, FOXA1-, GATA3-), CK5/6+ and GATA3- were significantly correlated with a poor OS when the CK5/6+ expression rate was $>10 \%$. CK5/6+ and FOXA1- were also significantly correlated with a poor OS and RFS when the CK5/6+ expression rate was $>10$ and $>25 \%$. When we defined the basal type according to the new consensus, we found a significant correlation with poor OS, and a tendency toward an association with RFS. This somewhat unclear correlation is likely due to the insufficient number of specimens analyzed in our study. However, this finding suggests a clear relationship between the basal type and a poor prognosis.

This study has some limitations. This study had a retrospective design, and the sample size was relatively small. Therefore, more extensive and prospective studies are needed to verify the observed associations. And we did not consider the number of TURBs or intravesical treatments that could affect the outcome. Nevertheless, it is meaningful that this study applied the newly established BASQ classification to the evaluation of clinical specimens from patients diagnosed with bladder cancer and related the BASQ classification to prognosis. We could also confirm that the basal and luminal types in the BASQ classification are closely related to patient prognosis.

\section{Conclusions}

In this study, CK14 expression was associated with a poor prognosis. The new classification system of bladder cancer based on molecular characteristics is expected to helpful tool for the establishment of personalized treatment strategies and associated prediction of therapeutic responses.

\section{Additional file}

Additional file 1: Figure S1. Nomogram for prediction of survival after cystectomy. (TIF $21 \mathrm{~kb}$ )

\section{Abbreviations}

Akt: protein kinase B; ASA: American society of anesthesiologists; BASQ: basal/squamous-like; FGFR3: fibroblast growth factor receptor-3; IHC: Immunohistochemistry; mTOR: mammalian target of rapamycin; PI3K: phosphatidylinositol-3-OH kinase; PLND: pelvic lymph node dissection; TCGA: the cancer genome atlas; TMA: tissue microarray; TP53: tumor protein 53; TURB: transurethral removal of the bladder tumor

\section{Author's contributions}

Conception and design JK, KM, HY, CJ, CK, HK acquisition of data HY, CJ, KM, JK analysis and interpretation of data HY, KM, JK drafting of the manuscript $\mathrm{HY}$ critical revision of the manuscript for important intellectual content $\mathrm{HY}$, KM, CJ, CK, HK, KM, statistical analysis HY, KM, JK obtaining funding JK 
administrative, technical, or material support CJ, CK, HK supervision KM, JK. All authors read and approved the final manuscript.

\section{Funding}

This study was supported by the National Research Foundation of Korea (NRF) grant funded by the Korea government (MSIP) (Grant number: 2016R1A2B4011623). No funders had any role in study concept and design, experiments, analysis of data, writing manuscript, or the decision for publication

This study was supported by the 2015 Korean Urologic Oncology Society Grant.

\section{Availability of data and materials}

The datasets used and/or analysed during the current study are available from the corresponding author on reasonable request.

\section{Ethics approval and consent to participate}

This study was approved by the Institutional Review Board of Seoul National University Hospital (IRB No. H-1806-081-951). We used the human bladder cancer materials stored in the cancer tissue bank of Seoul National University Hospital (IRB No. H1307-084-505). We obtained informed written consent from all research participants.

\section{Consent for publication}

Not applicable

\section{Competing interests}

The authors declare that they have no competing interests.

\section{Author details}

${ }^{1}$ Department of Urology, Inje University College of Medicine, Inje University Sanggye Paik Hospital, Seoul, Korea. ${ }^{2}$ Department of Urology, Seoul National Univervity College of Medicine, Seoul National University Hospital, Seoul, Korea. ${ }^{3}$ Department of Pathology, Seoul National Univervity College of Medicine, Seoul National University Hospital, Seoul, Korea.

Received: 7 May 2019 Accepted: 15 August 2019

Published online: 09 September 2019

\section{References}

1. Siegel RL, Miller KD, Jemal A. Cancer statistics, 2017. CA Cancer J Clin. 2017; 67(1):7-30. https://doi.org/10.3322/caac.21387.

2. Babjuk M, Burger M, Zigeuner R, Shariat SF, van Rhijn BW, Comperat $E_{,}$ et al. EAU guidelines on non-muscle-invasive urothelial carcinoma of the bladder: update 2013. Eur Urol. 2013;64(4):639-53. https://doi.org/1 0.1016/j.eururo.2013.06.003.

3. Burger M, Oosterlinck W, Konety B, Chang S, Gudjonsson S, Pruthi R, et al. ICUD-EAU international consultation on bladder cancer 2012: non-muscleinvasive urothelial carcinoma of the bladder. Eur Urol. 2013;63(1):36-44. https://doi.org/10.1016/j.eururo.2012.08.061.

4. Cancer Genome Atlas Research N. Comprehensive molecular characterization of urothelial bladder carcinoma. Nature. 2014;507(7492): 315-22. https://doi.org/10.1038/nature12965.

5. Choi W, Porten S, Kim S, Willis D, Plimack ER, Hoffman-Censits J, et al. Identification of distinct basal and luminal subtypes of muscle-invasive bladder cancer with different sensitivities to frontline chemotherapy. Cancer Cell. 2014;25(2):152-65. https://doi.org/10.1016/j.ccr.2014.01.009.

6. Hoadley KA, Yau C, Wolf DM, Cherniack AD, Tamborero D, Ng S, et al. Multiplatform analysis of 12 cancer types reveals molecular classification within and across tissues of origin. Cell. 2014;158(4):929-44. https://doi.org/1 0.1016/j.cell.2014.06.049.

7. Lindgren D, Frigyesi A, Gudjonsson S, Sjodahl G, Hallden C, Chebil G, et al. Combined gene expression and genomic profiling define two intrinsic molecular subtypes of urothelial carcinoma and gene signatures for molecular grading and outcome. Cancer Res. 2010;70(9):3463-72. https:// doi.org/10.1158/0008-5472.CAN-09-4213.

8. Rebouissou S, Bernard-Pierrot I, de Reynies A, Lepage ML, Krucker C, Chapeaublanc $E_{\text {, et }}$ al. EGFR as a potential therapeutic target for a subset of muscle-invasive bladder cancers presenting a basal-like phenotype. Sci Transl Med. 2014;6(244):244ra291. https://doi.org/10.1126/ scitranslmed.3008970.
9. Volkmer JP, Sahoo D, Chin RK, Ho PL, Tang C, Kurtova AV, et al. Three differentiation states risk-stratify bladder cancer into distinct subtypes. Proc Natl Acad Sci U S A. 2012;109(6):2078-83. https://doi.org/10.1073/ pnas.1120605109.

10. Cantiello F, Cicione A, Autorino R, Salonia A, Briganti A, Ferro M, et al. Visceral obesity predicts adverse pathological features in urothelial bladder cancer patients undergoing radical cystectomy: a retrospective cohort study. World J Urol. 2014;32(2):559-64. https://doi.org/10.1007/ s00345-013-1147-7.

11. Ferro M, De Cobelli O, Buonerba C, Di Lorenzo G, Capece M, Bruzzese D, et al. Modified Glasgow prognostic score is associated with risk of recurrence in bladder Cancer patients after radical cystectomy: a multicenter experience. Medicine (Baltimore). 2015;94(42):e1861. https://doi.org/10.1097/ MD.0000000000001861.

12. Ferro M, Di Lorenzo G, Vartolomei MD, Bruzzese D, Cantiello F, Lucarelli G, et al. Absolute basophil count is associated with time to recurrence in patients with high-grade $\mathrm{T} 1$ bladder cancer receiving bacillus CalmetteGuerin after transurethral resection of the bladder tumor. World J Urol. 2019. https://doi.org/10.1007/s00345-019-02754-2.

13. Ferro M, Vartolomei MD, Russo Gl, Cantiello F, Farhan ARA, Terracciano D, et al. An increased body mass index is associated with a worse prognosis in patients administered BCG immunotherapy for T1 bladder cancer. World J Urol. 2019;37(3):507-14. https://doi.org/10.1007/s00345-018-2397-1.

14. Damrauer JS, Hoadley KA, Chism DD, Fan C, Tiganelli CJ, Wobker SE, et al. Intrinsic subtypes of high-grade bladder cancer reflect the hallmarks of breast cancer biology. Proc Natl Acad Sci U S A. 2014;111(8):3110-5. https:// doi.org/10.1073/pnas.1318376111

15. Kim J, Akbani R, Creighton CJ, Lerner SP, Weinstein JN, Getz G, et al. Invasive bladder cancer: genomic insights and therapeutic promise. Clin Cancer Res. 2015;21(20):4514-24. https://doi.org/10.1158/1078-0432.CCR-14-1215.

16. Ho PL, Kurtova A, Chan KS. Normal and neoplastic urothelial stem cells: getting to the root of the problem. Nat Rev Urol. 2012;9(10):583-94. https:// doi.org/10.1038/nrurol.2012.142.

17. Perou CM, Sorlie T, Eisen MB, van de Rijn M, Jeffrey SS, Rees CA, et al. Molecular portraits of human breast tumours. Nature. 2000:406(6797):74752. https://doi.org/10.1038/35021093.

\section{Publisher's Note}

Springer Nature remains neutral with regard to jurisdictional claims in published maps and institutional affiliations.

Ready to submit your research? Choose BMC and benefit from:

- fast, convenient online submission

- thorough peer review by experienced researchers in your field

- rapid publication on acceptance

- support for research data, including large and complex data types

- gold Open Access which fosters wider collaboration and increased citations

- maximum visibility for your research: over $100 \mathrm{M}$ website views per year

At BMC, research is always in progress.

Learn more biomedcentral.com/submission 